Internist $2021 \cdot 62: 1256$

https://doi.org/10.1007/s00108-021-01175-x

Online publiziert: 4. Oktober 2021

(c) Springer Medizin Verlag GmbH, ein Teil von Springer Nature 2021

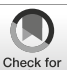

\section{Erratum zu: Pneumokokkenimpfstoffe}

\author{
Mathias W. Pletz ${ }^{1}$ Christina Bahrs, \\ ${ }^{1}$ Institut für Infektionsmedizin und Krankenhaushygiene, Universitätsklinikum Jena, Friedrich-Schiller- \\ Universität, Jena, Deutschland \\ ${ }^{2}$ Universitätsklinik für Innere Medizin I, Klinische Abteilung für Infektionen und Tropenmedizin, \\ Medizinische Universität Wien, Wien, Österreich
}

\section{Erratum zu: \\ Internist 2021 \\ https://doi.org/10.1007/s00108-021- \\ 01100-2}

InTab. 1 mit der Spalte Indikationsimpfung, Sonstige chronische Grunderkrankungen, Empfehlung der STIKO, Konjugatvakzine findet sich ein $X(1 \times)$. Dies ist nicht korrekt, da für eine chronische Grunderkrankung ohne Immunschwäche von der Ständigen Impfkommission (STIKO) nur die Polysaccharidvakzine empfohlen wird.

Der Originalbeitrag wurde korrigiert.

\section{Korrespondenzadresse}

Univ.-Prof. Dr. med. Mathias W. Pletz

Institut für Infektionsmedizin und Krankenhaushygiene, Universitätsklinikum Jena, Friedrich-Schiller-Universität Am Klinikum 1, 07747 Jena, Deutschland mathias.pletz@med.uni-jena.de
Die Online-Version des Originalartikels ist unter https://doi.org/10.1007/s00108-021-01100-2 zu finden.

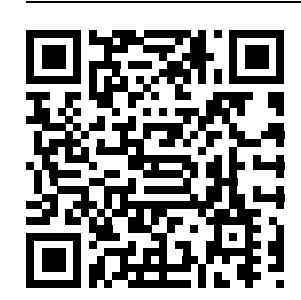

QR-Code scannen \& Beitrag online lesen 